\title{
Systematic study of operator dependence in nucleus calculation at large quark mass
}

\section{Takeshi Yamazaki ${ }^{\star a, b, c}$, Ken-Ichi Ishikawa ${ }^{d, c}$, Yoshinobu Kuramashi $^{b, a, c}$, Akira Ukawa ${ }^{c}$ for PACS Collaboration}

${ }^{a}$ Faculty of Pure and Applied Sciences, University of Tsukuba, Tsukuba, Ibaraki 305-8571, Japan

${ }^{b}$ Center for Computational Sciences, University of Tsukuba, Tsukuba, Ibaraki 305-8577, Japan

${ }^{c}$ RIKEN Advanced Institute for Computational Science, Kobe, Hyogo 650-0047, Japan

${ }^{d}$ Department of Physics, Hiroshima University, Higashi-Hiroshima, Hiroshima 739-8526, Japan.

Email: vamazakidhet.ph.tsukuba.ac.jp

\begin{abstract}
Recently it is claimed that there is a significant systematic error from excited state contributions in the nucleus correlation functions by comparing with calculations using the exponential and wall source operators. However, the wall source result is obtained in much earlier time than the plateau region. In order to investigate the systematic error in the plateau region, we calculate the correlation functions with both the operators in quenched QCD at $0.8 \mathrm{GeV}$ pion mass and in $N_{f}=2+1 \mathrm{QCD}$ at $0.7 \mathrm{GeV}$ pion mass in high accuracy. In this report we present preliminary results of those calculations, and show that the energy shift obtained from the two sources agree with each other, if those are determined from a region, where both the nucleon and two-nucleon correlation functions have plateaus.
\end{abstract}

34th annual International Symposium on Lattice Field Theory

24-30 July 2016

University of Southampton, UK

\footnotetext{
${ }^{*}$ Speaker.
} 


\section{Introduction}

The binding energies of light nuclei with the mass number less than or equal to four were

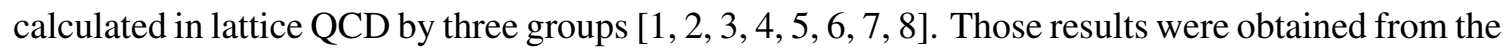
exponential or gaussian sources. Recently, HALQCD [Q] claimed a possibility of large systematic error coming from excited state contaminations, in other words source operator dependence, in the binding energy calculation. Therefore, in this study, we investigate source dependence of the energy shift in the spin-triplet two-nucleon channel using the exponential and wall sources.

In the binding energy calculation, the correlation functions for the nucleon $C_{N}(t)$ and twonucleon $C_{N N}(t)$ channels are calculated, and the energy shift between twice the nucleon mass and the ground state two-nucleon energy, $2 m_{N}-E_{N N}$, is evaluated from the ratio of the correlation functions, $C_{N N}(t) /\left(C_{N}(t)\right)^{2}$. An important condition of this analysis is that the energy shift must be determined from plateau regions of $C_{N}(t)$ and $C_{N N}(t)$. It is known that the wall source needs much larger $t$ than other smearing sources to obtain the ground state energy for the nucleon and multi-nucleon systems. Thus, we employ large quark masses, corresponding to $m_{\pi}=0.8 \mathrm{GeV}$ in $N_{f}=0$ and $0.7 \mathrm{GeV}$ in $N_{f}=2+1$, and also carry out huge number of measurement, more than $10^{5}$, of the correlation functions to obtain a signal in large $t$ region in the wall source calculation. Using the data in high accuracy for the exponential and wall sources, we study $t$ dependence of the effective energy shift in each source, and compare results for the two sources in each plateau region to examine whether these sources give different results or not. We also investigate volume dependence of the energy shift obtained from the wall source. All the results in this report are preliminary.

\section{Simulation parameters}

For the quenched QCD configuration, we employ Iwasaki gauge action at $\beta=2.416$, corresponding to $a=0.128 \mathrm{fm}[\mathbb{[}[\mathbf{0}]$. The quark propagators are calculated with a tad-pole improved Wilson action with $c_{\mathrm{SW}}=1.378$ at $\kappa_{u d}=0.13482$ corresponding to $m_{\pi}=0.8 \mathrm{GeV}$. The actions and parameters are the same as in our previous works [ [⿴囗十, []. The temporal lattice size is fixed to 64, while the spatial size is chosen to be 16,20 , and 32 .

For the $N_{f}=2+1$ QCD configuration, we use Iwasaki gauge and a nonperturbative $O(a)$ improved Wilson actions with the same parameters of $\beta$ and $c_{\mathrm{SW}}$ in Ref. [W], where $a=0.09 \mathrm{fm}$. We employ $\kappa_{u d}=0.1369425$ and $\kappa_{s}=0.1368530$ corresponding to $m_{\pi}=0.7 \mathrm{GeV}$. The lattice size is $L^{3} \times T=32^{3} \times 48$.

We calculate the correlation functions for the nucleon and two-nucleon spin-triplet channel using the exponential and wall sources with the point sink. In order to increase statistics, we carry out various measurements of the correlation functions in each configuration by changing the source spacetime position and source time slice for the exponential and wall sources, respectively.

\section{Result}

\section{1 $N_{f}=0$ QCD at $m_{\pi}=0.8 \mathbf{~ G e V}$}

Figure $\square$ presents twice the effective nucleon mass and the effective two-nucleon energy with 

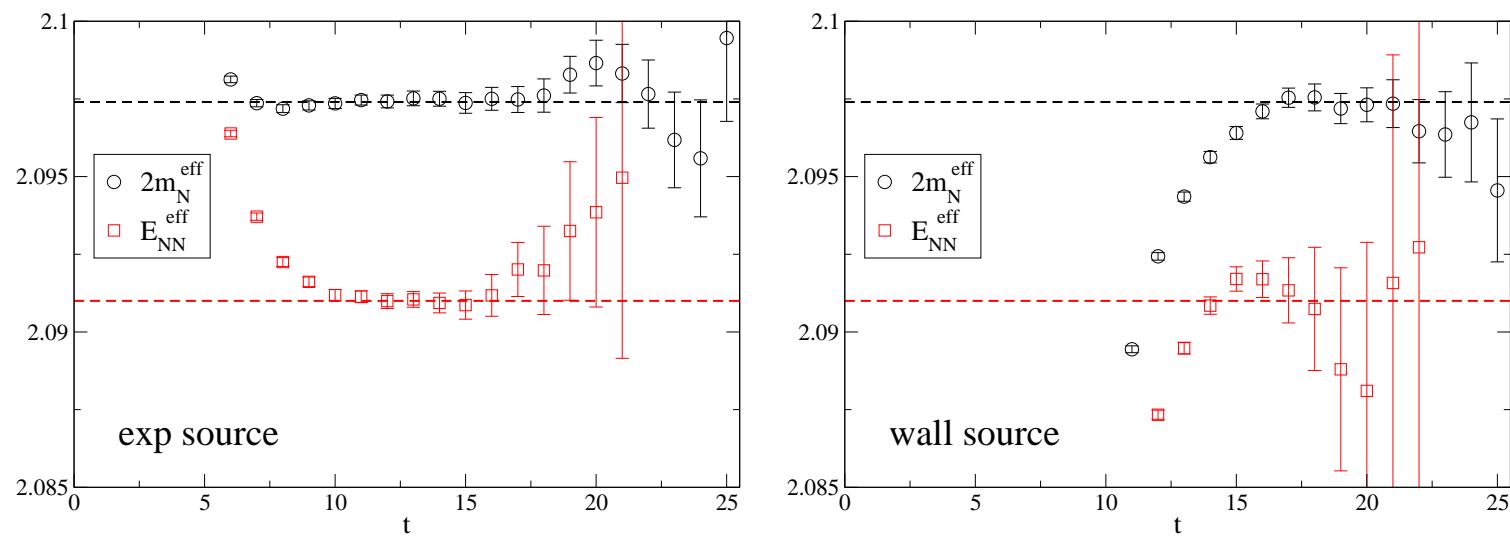

Figure 1: Effective nucleon mass $m_{N}^{\text {eff }}$ (circle) and two-nucleon energy $E_{N N}^{\text {eff }}$ (diamond) in the spin-triplet channel using the exponential (left panel) and wall (right panel) sources in the quenched case at $m_{\pi}=0.8$ $\mathrm{GeV}$. To compare $m_{N}^{\text {eff }}$ with $E_{N N}^{\text {eff }}, m_{N}^{\text {eff }}$ is multiplied by 2 in the figures. The dashed lines express the values of each plateau in the exponential source.

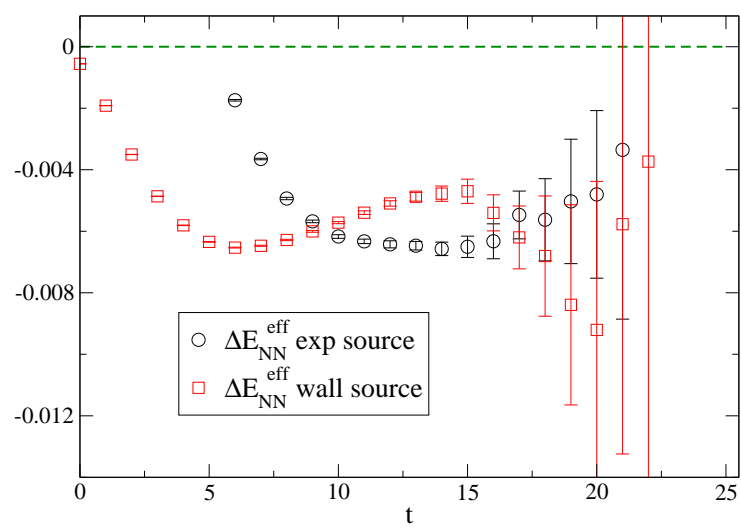

Figure 2: Effective energy shift $\Delta E_{N N}^{\mathrm{eff}}=2 m_{N}^{\mathrm{eff}}-E_{N N}^{\mathrm{eff}}$ using the exponential (circle) and wall (diamond) sources in the quenched case at $m_{\pi}=0.8 \mathrm{GeV}$.

the exponential (left panel) and wall (right panel) sources on $L=20$. The number of the measurement is about $5 \times 10^{6}$ in both the sources. The results of the exponential source have reasonable plateaus, which start from $t \sim 11$. The dashed line expresses each value in the plateau region of the exponential source. The results of the wall source have larger errors in the plateau region than the exponential one due to the late plateau, which start from $t \sim 16$. The effective mass and energy of the wall source in the plateau region are reasonably consistent with the ones obtained from the exponential source.

The effective energy shift, which is evaluated from the ratio of the correlation functions $C_{N N}(t) /\left(C_{N}(t)\right)^{2}$, is plotted in Fig. ‥ A reasonable plateau is seen in the result of the exponential source, whose region is consistent with the plateau region of each correlation function shown in the left panel of Fig. $\mathrm{W}$. On the other hand, the result of the wall source does not have a plateau in $t<15$. The reason is that both the correlation functions in the ratio do not reach plateaus in this region as shown in the right panel of Fig. $⿴$. In the plateau region of each correlation function, $t \gtrsim 16$, the result of the wall source agrees with the plateau value of the exponential source within 

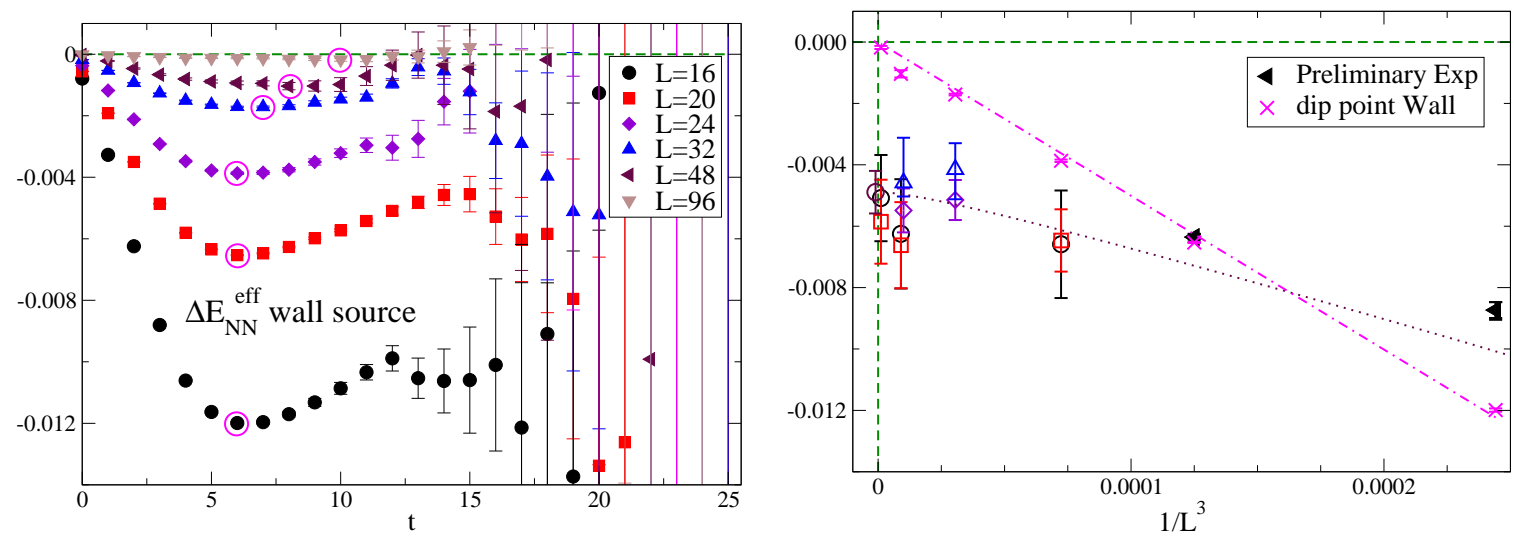

Figure 3: The effective energy shift $\Delta E_{N N}^{\text {eff }}$ using the wall source on several volumes (left panel) and volume dependence of the energy shift (right panel). In the left panel, the data on $L=16,20,32$ are preliminary results in the current calculation, while those on $L=24,48,96$ are the ones in pilot studies for the previous work [目]. In the right panel, the cross symbols are the data of the dip point expressed by the open circle in the left panel, and the dash-dot line is for the guide of eye. The open symbols and dashed line are the results in the previous works [ [ [] . The closed triangle represents a preliminary result obtained from the exponential source in the current calculation.

the large error.

From the comparison, we conclude that a consistent energy shift is obtained from the exponential and wall sources, when those values are determined from each plateau region. Furthermore, the wall source needs much larger statistics than the exponential source to obtain a clear signal of the energy shift.

The volume dependence of the effective energy shift using the wall source is plotted in the left panel of Fig. 目, which includes the data of this work $(L=16,20,32)$ and also the ones of a pilot study of the previous work [ [ $[$ ] $(L=24,48,96)$. In small $t$ region the effective energy shift largely depends on the volume, and increases with the volume. The results in all the volumes have a dip in the region. If statistics is not enough, one might regard the dip as a plateau, especially in larger volume, because the $t$ dependence is milder. When we pick up the dip point in each volume, which is the minimum value in the small $t$ region as expressed by open circles in the figure, they roughly behave as $1 / L^{3}$ as presented in the right panel of Fig. [1]. This behavior is consistent with the one observed by HALQCD using the wall source in Ref. [Q]. This, however, is not the volume dependence of the ground state energy shift, because it must be determined from the plateau region, which is roughly more than 15 in our wall source data as shown in Fig. 1 . In our data, the effective energy shift has large error in the region, and it is hard to estimate a precise energy shift from the wall source data. On the other hand, from the exponential source the ground state energy shifts can be determined precisely as shown in the right panel of Fig. [3, whose volume dependence is reasonably consistent with the one of the previous work [ [D].

\section{$3.2 N_{f}=2+1$ QCD at $m_{\pi}=0.7 \mathrm{GeV}$}

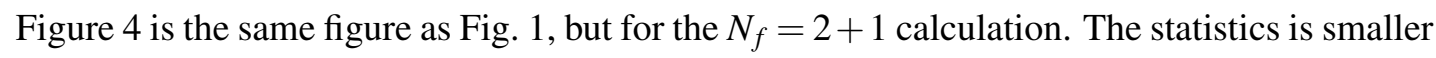
than the ones in the quenched case. The numbers of the measurement for the exponential and wall 

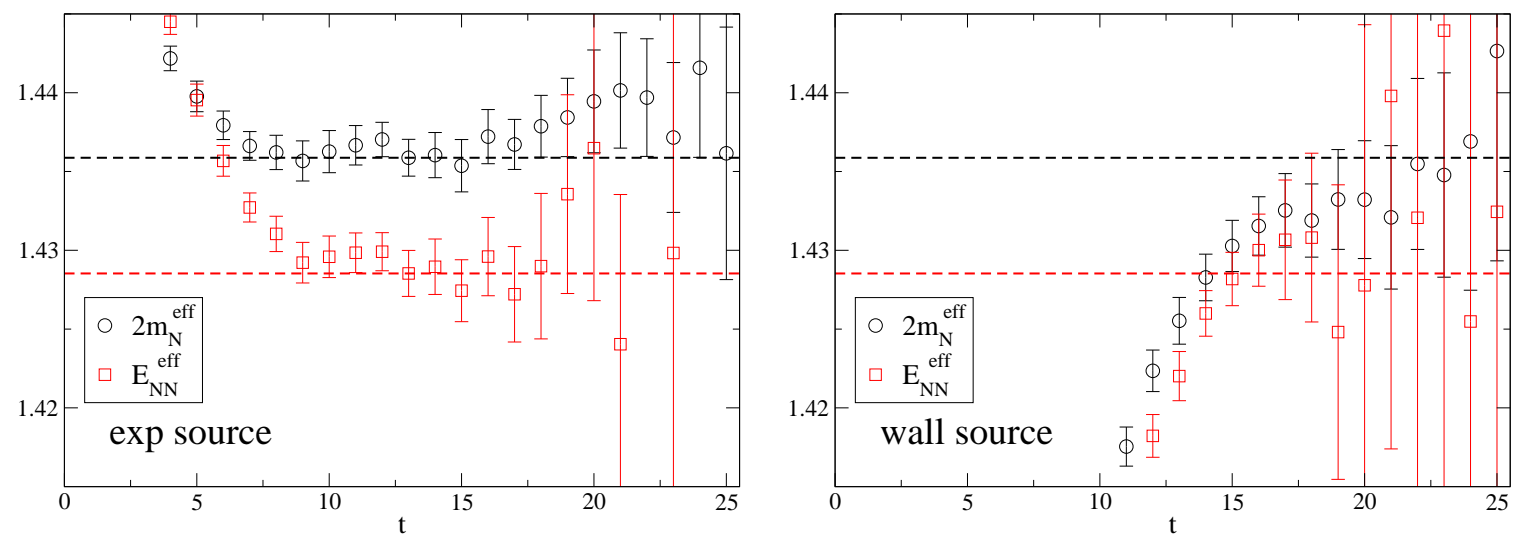

Figure 4: The same figure as Fig. 囬, but for $N_{f}=2+1$ calculation at $m_{\pi}=0.7 \mathrm{GeV}$.

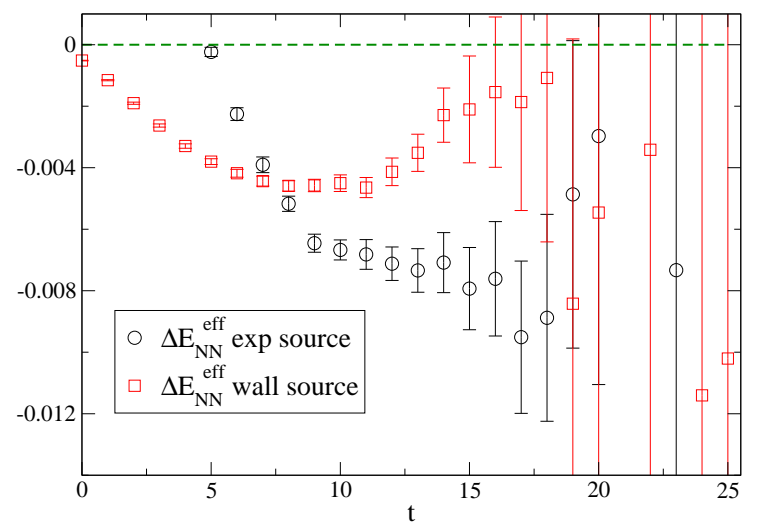

Figure 5: The same figure as Fig. 凹, but for $N_{f}=2+1$ calculation at $m_{\pi}=0.7 \mathrm{GeV}$.

sources are $2 \times 10^{5}$ and $1 \times 10^{5}$, respectively. As in the quenched case, the data of the exponential source have plateau, which start from $t \sim 9$. For the wall source, it is unclear where plateaus start due to larger statistical error. The effective nucleon mass seems to have a plateau in the region $t \gtrsim 16$, while the value of the effective mass is smaller than the one of the exponential source. When the statistics is increased, the plateau of the wall source will appear in a larger $t$ region, $t \gtrsim 20$, and its value will agree with the result of the exponential source.

The effective energy shifts from the two sources are plotted in Fig. [1. The result of the exponential source has a reasonable plateau in the region, where the effective mass and energy have their plateau as shown in the left panel of Fig. $\theta$. In the wall source a $t$ independent behavior is seen in $t=8-11$, although the effective mass and energy do not reach a plateau in this region. This is a similar behavior to the dip structure as seen in the quenched data. Since the lattice spacing in $N_{f}=2+1$ is finer than the one in the quenched case, the dip structure becomes wider in the $N_{f}=2+1$ case. The $t$ independent behavior would be regarded as a plateau, if the statistics is not enough. In such a case, one might conclude that different results are obtained from the two sources. However, the energy shift of the ground state must be determined from the plateau region of each correlator in the right panel of Fig. 田. Unfortunately, our data do not have enough statistics to obtain the effective energy shift clearly in this region, while the value in $t \gtrsim 20$ is consistent with 
the one obtained from the exponential source within the large error of the wall source. In order to check the consistency further, it is important to increase statistics by generating configurations.

\section{Summary}

We have investigated source operator dependence of the energy shift in the spin-triplet twonucleon channel in the $N_{f}=0$ and $N_{f}=2+1$ cases. From the comparisons with the results with the exponential and wall sources, it is concluded that the two sources give consistent results in each plateau region, when the statistics is large enough. Using the exponential source, the energy shift can be determined from the plateau regions for the nucleon and two-nucleon correlation functions, while from the wall source it is hard to obtain the energy shift in a similar quality to the exponential source due to the late plateaus for the correlation functions.

It is also concluded that the effective energy shift in the wall source always has the dip structure in the small $t$ region, where the nucleon and two-nucleon correlation functions do not reach a plateau. If the statistics is not enough, the dip structure might look like a plateau, especially in a fine lattice spacing on a large volume. In such a case, the energy shift, which is determined from the dip point, roughly behaves as $1 / L^{3}$. This is a consistent behavior with the wall source result obtained by HALQCD Collaboration in Ref. [Q].

We consider that the properties observed in the current study at the large $m_{\pi}$ will be seen in

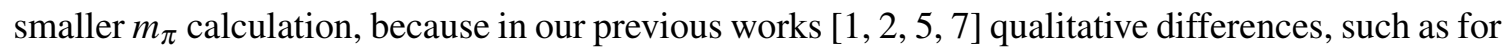
the existence of the light nuclei, were not observed in $m_{\pi}=0.3-0.8 \mathrm{GeV}$. Although the consistency between the exponential and wall sources is investigated in this calculation, more reliable results can be obtained from the method using the generalized eigenvalue problem [ए2]. Therefore, it is an important future work to calculate the two-nucleon energy using the more reliable method, and compare the energy of the ground state using the method with those obtained from plateau regions of the exponential and wall sources.

\section{Acknowledgements}

Numerical calculations for the present work have been carried out on the FX10 supercomputer system at Information Technology Center of the University of Tokyo, on the COMA cluster system under the "Interdisciplinary Computational Science Program" of Center for Computational Science at University of Tsukuba, on the Oakforest-PACS system of Joint Center for Advanced High Performance Computing, on the computer facilities of the Research Institute for Information Technology of Kyushu University, and on the FX100 and CX400 supercomputer systems at the Information Technology Center of Nagoya University. This research used computational resources of the HPCI system provided by Information Technology Center of the University of Tokyo through the HPCI System Research Project (Project ID: hp160125). We thank the colleagues in the PACS Collaboration for providing us the code used in this work. This work is supported in part by Grants-in-Aid for Scientific Research from the Ministry of Education, Culture, Sports, Science and Technology (Nos. 25800138, 16H06002). 


\section{References}

[1] PACS-CS Collaboration, T. Yamazaki, Y. Kuramashi, and A. Ukawa, Phys.Rev. D81 (2010) 111504.

[2] PACS-CS Collaboration, T. Yamazaki, Y. Kuramashi, and A. Ukawa, Phys. Rev. D84 (2011) 054506.

[3] NPLQCD Collaboration, S. Beane et al., Phys. Rev. D85 (2012) 054511.

[4] NPLQCD Collaboration, S. Beane, E. Chang, S. Cohen, W. Detmold, H. Lin, et al., Phys.Rev. D87 (2013), no. 3034506 .

[5] T. Yamazaki, K.-i. Ishikawa, Y. Kuramashi, and A. Ukawa, Phys.Rev. D86 (2012) 074514.

[6] CalLat Collaboration, E. Berkowitz, T. Kurth, A. Nicholson, B. Joo, E. Rinaldi, M. Strother, P. M. Vranas, and A. Walker-Loud, Phys. Lett. B765 (2017) 285-292.

[7] T. Yamazaki, K.-i. Ishikawa, Y. Kuramashi, and A. Ukawa, Phys. Rev. D92 (2015), no. 1014501.

[8] NPLQCD Collaboration, K. Orginos, A. Parreno, M. J. Savage, S. R. Beane, E. Chang, and W. Detmold, Phys. Rev. D92 (2015), no. 11114512.

[9] HALQCD Collaboration, T. Iritani et al., JHEP 10 (2016) 101.

[10] CP-PACS Collaboration, A. Ali Khan et al., Phys. Rev. D65 (2002) 054505.

[11] PACS-CS Collaboration, S. Aoki et al., Phys. Rev. D81 (2010) 074503.

[12] M. Lüscher and U. Wolff, Nucl. Phys. B339 (1990) 222-252. 